\title{
BMJ Open Is the incidence of heart attack still decreasing in Australia? Developing reliable methods for monitoring trends in myocardial infarction and coronary heart disease (AUS-MOCHA): a study protocol
}

\author{
Lee Nedkoff, ${ }^{1}$ Matthew Knuiman, ${ }^{1}$ Michael S T Hobbs, ${ }^{1}$ Joseph Hung, ${ }^{2}$ \\ Sushma Mathur, ${ }^{3}$ John Beilby, ${ }^{4,5}$ Anna Reynolds, ${ }^{3}$ Tom G Briffa, ${ }^{1}$ Derrick Lopez, ${ }^{1}$ \\ Frank M Sanfilippo ${ }^{1}$
}

To cite: Nedkoff L, Knuiman M, Hobbs MST, et al. Is the incidence of heart attack still decreasing in Australia? Developing reliable methods for monitoring trends in myocardial infarction and coronary heart disease (AUS-MOCHA): a study protocol. BMJ Open 2016;6:e12180. doi:10.1136/bmjopen-2016012180

- Prepublication history for this paper is available online. To view these files please visit the journal online (http://dx.doi.org/10.1136/ bmjopen-2016-012180).

Received 6 April 2016 Revised 30 June 2016 Accepted 1 August 2016

CrossMark

For numbered affiliations see end of article.

Correspondence to

Dr Lee Nedkoff;

lee.nedkoff@uwa.edu.au

\section{ABSTRACT}

Introduction: Accurate monitoring of acute coronary heart disease (CHD) is essential for understanding the effects of primary and secondary prevention and for planning of healthcare services. The ability to reliably monitor acute CHD has been affected by new diagnostic tests for myocardial infarction (MI) and changing clinical classifications and management of CHD. Our study will develop new and reliable methods for monitoring population trends in incidence, outcomes and health service usage for acute CHD and chest pain.

Methods and analysis: The study cohort of all CHD will be identified from the Western Australian Data Linkage System using state-wide data sets for emergency department presentation, hospitalisations and mortality data for 2002-2014. This core linked data set will be supplemented with data from hospital medical record reviews, pathology data and hospital pharmacy dispensing databases. The consistency over time of the coding of the different subgroups of $\mathrm{CHD} /$ chest pain (ST-elevation MI, non-ST elevation MI, unstable angina, stable angina, other $\mathrm{CHD}$, non-CHD chest pain) in linked data will be assessed using these data sources, and an algorithm developed detailing groups in which temporal trends can be reliably measured. This algorithm will be used for measurement of trends in incidence and outcomes of acute $\mathrm{CHD}$, and to develop further methods for monitoring acute $\mathrm{CHD}$ using unlinked and linked data with varying availability of hospitalisation history.

Ethics and dissemination: Ethics approval has been obtained from the Human Research Ethics Committees of the WA Department of Health (\#2016/23) and The University of Western Australia (RA/4/1/7230). Findings will be disseminated via publication in peer-reviewed journals, and presentation at national and international conferences. There will also be a strong platform for dissemination of new monitoring methods via collaboration with the Australian Institute of Health and
Strengths and limitations of this study

- This whole-population linked data study will develop new methods for monitoring coronary heart disease and will include linkage of cardiac biomarker and clinical data to hospitalisation, mortality and emergency department data sets.

- Algorithms will be developed which can be applied across linked and unlinked data systems to measure temporal trends in coronary heart disease. This will allow translation of methods to state-based and national monitoring systems, and allow improved use of linked data in epidemiological studies.

- Linked administrative data will be used for the whole-population analyses within the study, and thus, the limitations of these data sources could impact on accuracy. However, analyses using clinical and biomarker data in smaller patient samples will guide methods for the wholepopulation analyses.

Welfare which will assist with promotion of these methods at state and national levels.

\section{INTRODUCTION}

While mortality from coronary heart disease (CHD) has been declining in developed countries including Australia for 40 years, ${ }^{1}$ CHD remains the leading cause of death in Australia and the leading contributor to the burden of disease. ${ }^{2}$ Acute CHD events are still life-threatening and require rapid and expensive healthcare interventions. Accurate information on trends in acute CHD event rates is essential for appropriate planning and evaluation of public health and clinical 
services. Until the late 1990s, it was possible to monitor the incidence of acute CHD events using hospitalisation and mortality data. ${ }^{3}$ However, there are increasing concerns about the utility of this method because of attenuation in the long-term decline in trends in MI admissions since the late 1990s. ${ }^{4}{ }^{5}$ Reasons for this include the changing clinical diagnosis of MI, the introduction of new highly sensitive and specific biomarkers of myocardial damage (troponins) and increasing hospital presentations for suspected MI, but the effects on population-level monitoring have not been quantified in Australia or in many other jurisdictions.

The clinical definition of MI was redefined internationally in the late 1990s with the addition of troponin tests to the clinical algorithm for detecting MI. ${ }^{6}$ Patients presenting with elevated troponin levels in the context of myocardial ischaemia, even with normal levels of creatine kinase (CK), were diagnosed as MI, and this is now incorporated into clinical guidelines for the management of patients with suspected acute coronary syndromes (ACS). ${ }^{7}$ Troponin tests have increased the detection of non-ST elevation MI (NSTEMI $)^{5} 8$ and have arguably increased counts of MI in administrative data at the expense of other categories of CHD, including stable and unstable angina. However, there have been no studies in Australia on the consistency of coding over time for unstable and stable angina, nor of ST segment elevation MI (STEMI) and NSTEMI, and the reliability of these hospital discharge diagnoses for trend analysis is unknown.

While clinicians now diagnose MI in terms of STEMI and NSTEMI, International Classification of Diseases (ICD) codes have until recently been restricted to the pathological diagnosis of transmural or subendocardial MI using a fourth digit code. A reference to STEMI ('transmural or STEMI') and NSTEMI ('subendocardial or NSTEMI') was added to the description of these codes in the Australian revision of ICD-10 (ICD-10-AM) in 2004, and to ICD-9 in the USA in $2005 .{ }^{9}$ Although STEMI is indicative of acute transmural injury, ECG diagnostic criteria for transmural MI may not be synonymous with STEMI. Nevertheless, the fourth digit ICD code for MI has been used to demonstrate downward trends in STEMI and increases in NSTEMI. ${ }^{10}{ }^{11}$ However, there remains a lack of published evidence on how well these codes in administrative data correlate with the STEMI/NSTEMI diagnosis. Given the importance of this clinical classification in guiding treatment and management, detailed research is required to establish the reliability of fourth digit codes for STEMI and NSTEMI in administrative data.

Other changes in CHD treatment and presentation also complicate approaches to monitoring. Marked increases in rates of percutaneous coronary intervention have occurred, due largely to adoption of an early invasive strategy in high-risk ACS according to national guidelines. ${ }^{7}$ Additionally, presentations to the emergency department (ED) and hospitalisations for non-CHD chest pain have increased nationally, ${ }^{12}$ with $>80 \%$ increase recorded in Perth from 1998 to 2008, and there are little data characterising this increasing patient group. This highlights the need to investigate the utility of incorporating ED data in monitoring algorithms.

Accurate monitoring of trends is important for identifying changes in the epidemiology of a disease and demonstrating the impacts of acute care and primary and secondary prevention at the population level. The use of linked hospital morbidity data makes it possible to identify incident and recurrent CHD events, comorbidities, hospital transfers, case fatality and define person-based cohorts and follow-up events for outcome studies. ${ }^{4}{ }^{13-16}$ Using unlinked data for these purposes leads to overcounting and possible temporal and geographical biases. Previous methods for measuring the incidence of major coronary events incorporating length of stay, 28-day episode of care and admission type ${ }^{3}$ have now become less relevant. In Australia, national monitoring of CHD is undertaken by the Australian Institute of Health and Welfare (AIHW) primarily using unlinked data. Validation work by the AIHW demonstrates that the current monitoring algorithm using unlinked data underestimates ACS events by $6 \%$ in Western Australia (WA) and $11 \%$ in New South Wales (NSW). ${ }^{17}$ WA has had a systematic record linkage system in place for two decades, and other states and territories in Australia now have the infrastructure for record linkage, although are at different stages of development and implementation. The need for methods which will provide reliable trend estimates across multiple jurisdictions is therefore imperative.

\section{Aims}

The AUS-MOCHA project will aim to develop new and reliable methods for monitoring population trends in incidence, outcomes and health service utlisation for acute CHD and chest pain. The specific research objectives are:

(1) To determine the consistency of coding over time in hospital morbidity data for subgroups of CHD hospital presentations (STEMI/NSTEMI, unstable angina, stable angina, other CHD) and chest pain, and define subgroups and aggregated groups that permit reliable analysis of trends in admissions and outcomes;

(2) To examine trends in incidence, clinical outcomes and health service utilisation using a new monitoring algorithm for the defined subgroups of acute CHD and chest pain;

(3) To develop and implement methods for monitoring trends in acute CHD for use in a variety of administrative data settings, including in unlinked data and linked data with limited available history.

\section{METHODS}

\section{Study design}

The AUS-MOCHA study is a retrospective linked data study, using whole-population administrative health data, supplemented with clinical and laboratory data sets. 


\section{Study setting and data source}

The population of WA was 2.6 million in 2015, comprising $10.9 \%$ of the Australian population. ${ }^{18}$ The majority of the WA population reside in the Perth metropolitan area $(79 \%)$. WA has been reported as being representative of national major sociodemographic and health indicators, including median age, proportion living in rural or remote areas, proportion of low-income households and proportion of the population whom are Indigenous $(3 \%) .{ }^{19}$

The Australian healthcare system is a mixed publicprivate system, managed by state and national governments. Statutory requirements in WA ensure reporting of minimum data requirements for all hospitalisations and deaths. All public and all private hospital presentations and admissions are captured within the state health data systems. Of the major adult hospitals in the Perth metropolitan area which receive acute CHD presentations, seven $(63 \%)$ are public, including the three major teaching hospitals. There are two major private hospitals in regional areas in WA, with the remainder of the state serviced by regional public hospital and health services. Diagnostic, interventional and surgical management of CHD patients is centralised in the metropolitan area, often necessitating transfer of patients over long distances.

The core data sets for this study will be obtained from the long-standing Western Australian Data Linkage System (WADLS) which contains population-level administrative health data for the entire state, held by the WA Department of Health. The system has been extensively used for epidemiological health research studies, including cardiovascular disease. The WADLS centrally links core data sets using a well-established method of probabilistic matching, based on key demographic variables, including name, address, date of birth and gender. Uncertain links are checked manually, and the accuracy of the linkage has been reported at $>99 \% .{ }^{20}$ Because of statutory and minimum data requirements, the core data sets of the WADLS have complete population coverage.

This study will establish a file of linked records based on three of the core WADLS data sets-Hospital Morbidity Data Collection (HMDC), Mortality register and Emergency Department Data Collection (EDDC). The HMDC provides coverage of all inpatient hospitalisations in WA across the public and private systems. Variables contained within the system include demographic details, admission and discharge dates, principal and up to 20 secondary discharge diagnosis fields, all inpatient procedures, admission type (emergency/ elective), separation type (indicating transfer or discharge) and hospital location.

The EDDC data set includes referral source, presenting symptom, major diagnostic category, departure destination and principal discharge diagnosis. The mortality data set contains date and cause of death, with cause of death coding in Australia following the recommended format of the WHO. ${ }^{21}$ Underlying cause of death is available for the whole data set. Multiple cause of death coding was adopted in Australia in 1997 and includes all conditions recorded on the death certificate, including underlying, associated and contributory causes of death. Full state coverage of the HMDC and mortality data sets are available from 1980 onwards, and the EDDC from 2002 onwards. The discharge diagnoses in the HMDC and cause of death coding in the mortality data set are coded using ICD codes, while in the EDDC data set, they are identified from text fields and symptom codes. In WA, records prior to 30 June 1999 are coded using the ICD-9 revision, and records subsequent to this date using ICD-10-Australian modification (AM). The Electoral Roll is one of the core data sets of the WADLS, and data from the roll will be linked to the core study data set for all people included in the study cohort. The Electoral Roll data set contains flags indicating whether a person is on the electoral roll, out of state or not on the electoral roll. These status flags are updated when the Electoral Commission is notified of changes, and the data set can be used as an indicator of location or proxy for checking prevalence status. Full state coverage is available from 1988 onwards.

The whole-population linked data set will be merged with hospital pharmacy dispensing data, available from all hospitals. We will also obtain results of cardiac biomarker tests for all patients in WA who received a cardiac biomarker test (troponin and/or CK or CK-MB) between 2000 and 2013. These will be available electronically from public and private pathology databases. These supplementary data sets will be linked to the core whole-population data set using a unique medical record number.

\section{Study period and cohort}

The linked data set will include records for anyone with a $\mathrm{CHD} /$ chest pain hospitalisation, ED presentation or death in WA between 1980 and 2014, as well as all linked hospital, ED and death records for this cohort. We will then identify any record within the core linked data set for the following CHD/chest pain subgroups: STEMI, NSTEMI, unstable angina, stable angina, other CHD and chest pain. We will also identify a subcohort of patients according to the hierarchy shown in table 1 for the period 20022014. This will include people aged $\geq 35$ years residing in WA (based on postcode and electoral roll information) at the time of the CHD event. Previous studies have used an upper age limit of 84 years because of known decreasing sensitivity for the recording of MI in hospital morbidity data with increasing age. ${ }^{22}$ Our initial age range will be 35-84 years, but our analyses will also investigate ages above 84 years and the utility and significance of these older age groups in analyses of trends and in identifying clinical subgroups of CHD. Unspecified MI (I21.9) will also be included in a sensitivity analysis.

\section{Covariates and clinical outcomes}

Comorbidities will be identified using a fixed period of linked hospitalisation history of up to 15 years for each 
Table 1 Identification of the study cohort using hospital morbidity data, emergency department data and mortality data for 2002-2014

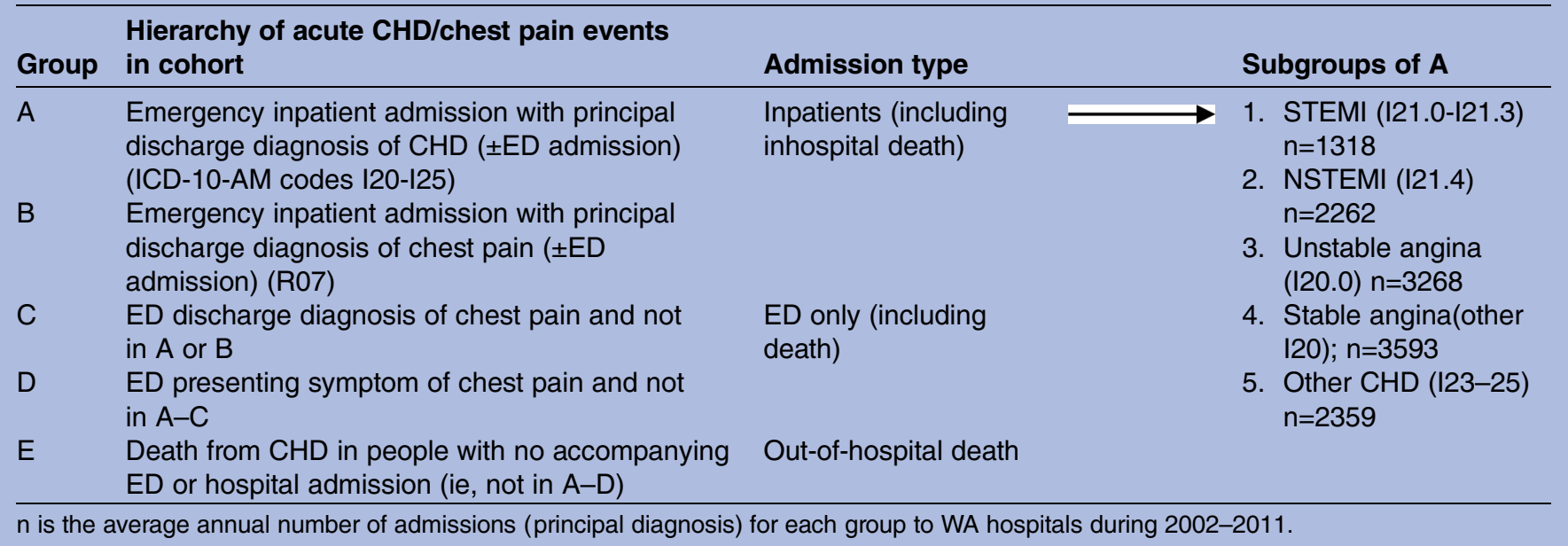

person in the cohort. The comorbidities of interest amenable to identification using hospitalisation data include hypertension, diabetes, heart failure, atrial fibrillation, prior stroke, peripheral arterial disease and chronic kidney disease. Data on prior CHD, invasive treatment (cardiac stents or surgery) and conditions associated with potential false-positive cardiac biomarker results (eg, Angioplasty, cardiac surgery, other major surgery, arrhythmias, heart failure, pulmonary embolism, renal failure, trauma, sepsis) will also be derived from the linked data set.

Outcomes will be derived from hospital and death data within the core linked data set. Complete follow-up for the cohort will be available to 31 December 2014, allowing for maximum follow-up of 13 years. Outcomes of interest will be recurrent MI, other acute CHD, readmission for coronary angiography or revascularisation procedures and death (cardiovascular and all-cause).

\section{Classification of cardiac biomarkers}

Classification of biomarkers will be based on the reference levels at each relevant hospital in the year of admission. Troponin results will be coded as positive, normal, equivocal or missing, ${ }^{23}$ and CK results coded as positive, normal or missing. Biomarkers will be grouped as none, CK only, troponin only, or troponin and CK. In WA, CK was recorded in conjunction with troponin up to the end of 2013, and therefore, CK and troponin results will be available in the majority of cases.

\section{Analysis}

Objective 1: Consistency of coding in hospital morbidity data for defined subgroups of acute CHD

The temporal consistency of CHD codes in administrative data will be evaluated, and based on the findings, an algorithm developed to identify subgroups of CHD within administrative data that are reliable for monitoring purposes. This will be an iterative process, with three major steps:
1. Determining trends: There are six CHD/chest pain subgroups that can be directly identified from HMDC records (STEMI, NSTEMI, unstable angina, stable angina, other CHD, chest pain). Consistency over time in the composition of each of these six subgroups in terms of the distribution of clinical and service characteristics of the cases will be assessed. The characteristics that are available from the core linked data set include: discharge diagnosis field (principal vs secondary), length of stay, admission type (emergency/elective), 28-day episodes, ED presentation, hospital type and location, coronary procedure, history of CVD admissions and cardiac biomarkers. CHD cases identified from the HMDC will be stratified using the biomarker classification detailed above (no positive biomarkers, CK-positive only, troponin-positive only, CK and troponinpositive). Our previous research has shown differing trends in rates of MI measured from the HMDC compared with various biomarker algorithms up to $2003{ }^{22}$ This analysis will extend these trend data and determine whether rates of different biomarker classifications to define MI have continued to diverge. Consistency of the characteristics over time within CHD/chest pain subgroups will be assessed using regression models (eg, logistic regression for proportion of cases with emergency admission type) and will be conducted separately for men and women, and age-adjusted or analysed by age strata.

2. Hospital record reviews: Some important characteristics of cases are not available in the core linked data set and the consistency over time in the distribution of these additional characteristics within each of the six subgroups will be based on random samples of cases in 2003, 2008 and 2013. Hospital medical records will be reviewed for these cases to obtain the additional characteristics, including chest pain on admission (typical or atypical including nausea, pallor, sweating, collapse, breathlessness)), acute 
complications (cardiogenic shock, heart failure), cardiac biomarkers (days 1-3) and ECGs (first ECG on admission, days 2-3, last ECG of admission).

The data collected from hospital records will be used to investigate the reliability and consistency over time of the fourth digit ICD MI code for distinguishing STEMI and NSTEMI in the administrative hospital data. ECGs collected for the random sample of MI patients will be reviewed by two cardiologists. The American Heart Association criteria for the management of STEMI ${ }^{24}$ and NSTEMI/unstable angina ${ }^{25}$ will be used to classify indicative ECG changes. We will calculate the observed agreement between the discharge diagnosis codes for STEMI/NSTEMI in the administrative data versus a combined classification of MI type from ECG findings (derived from the hospital record reviews), biomarker classifications and symptoms, taking into account potential causes of false negatives. The positive predictive value (PPV) is the proportion of HMDC-based cases of a particular type (eg, STEMI) that are confirmed via data from medical notes to be that type of case. A PPV of around $90 \%$ based on a sample size of 200 has an SE of $2 \%$. Thus, our goal is to sample 200 HMDC-based cases of STEMI and 200 cases of NSTEMI in each of 2003, 2008 and 2013. Depending on the findings from step 1 , we may also review hospital records for random samples of cases from the other subgroups (unstable angina, stable angina, other CHD, chest pain).

3. Algorithm development: Algorithms based on linked administrative data that identify the different aggregations of the six CHD subgroups (and administratively definable subsets) which have been shown in analyses in steps 1 and 2 to provide the most consistent groups of cases will be developed. The groups to be further considered in Objective 2 will also include the hierarchical groupings shown in table 1 , and also predefined subgroups of clinical interest such as STEMI, ACS and biomarker +ve MI (cases coded as MI in the HMDC that have a positive CK test result from laboratory data). We will include out-of-hospital CHD deaths (Group E) in the development of the algorithms to test consistency of total MI/CHD incidence, and will also analyse out-of-hospital deaths as a separate group, including identification of prior hospitalisation history where present. Analyses will be carried out using underlying cause of death only and multiple cause of death coding.

\section{Objective 2: Trends in incidence, clinical outcomes and} health service usage for defined subgroups of acute CHD and chest pain utilisation developed algorithms

Trends in the incidence of total acute CHD hospitalisations, as well as each of the algorithm-defined subgroups (Objective 1), will be analysed for the whole population of WA from 2002 to 2014. Incident cases in each study year will be defined as those with no hospitalisation for $\mathrm{CHD}$ in the previous 22 years. Incidence rates by sex, 5-year age group and calendar year will be calculated for each algorithm-based subgroup. Whole-population counts for WA, stratified by calendar year, sex and 5-year age group, are available from the Australian Bureau of Statistics and will be used in estimating rates. They are based on actual counts in census years (every 5 years), as estimated counts for intermediate years and as projected counts for years since last census. Poisson regression models will be fitted to estimate the annual rate of change after adjustment for age, separately by sex and overall. There will be more than $90 \%$ power to detect a rate ratio of 1.2 over 13 years from 2002 to 2014 (equivalent to an annual rate of change of $1.015 \%$ or $1.5 \%$ ).

Outcomes following incident hospitalisation for each of the algorithm-defined groups will be measured. Thirty-day outcomes in each subgroup will be measured for men and women separately, and longer term outcomes (1, 2 and 5 years) estimated in 30-day survivors using Kaplan-Meier survival analysis. The Cox proportional hazards regression models stratified by sex will be used to compare trends in outcomes between each of the different CHD groups, adjusted for age, comorbidities (diabetes, heart failure, renal disease, hypertension, prior vascular disease), Aboriginal status, socioeconomic status, hospital type, admission type, coronary artery revascularisation procedures at initial admission and possibly a term for the introduction of troponin effect. There will be high statistical power to detect trends and differences in outcomes across groups, for example, two groups each with 1000 cases annually provide over $90 \%$ power to detect a $3 \%$ difference in 1-year or 5-year outcomes for that year, and when aggregated over the study period, provide $90 \%$ power to detect differences as small as $1 \%$.

Proportions and rates of angiography and coronary revascularisation procedures occurring within 30 days of onset of incident events will be calculated for total acute CHD and for each of the CHD subgroups. This will provide data on whether investigations and procedures are targeted appropriately at high-risk cases. Multivariable-adjusted logistic regression models will be used to examine temporal trends that are adjusted for demographics, clinical history and indicators, including time to angiography or revascularisation, based on methods used in administrative data. ${ }^{26}$

\section{Objective 3: Translation of evidence-based methods for monitoring trends in acute CHD using unlinked and linked health data}

The findings from objectives 1 and 2 will be used to develop further methods for the monitoring of acute $\mathrm{CHD} /$ chest pain for use in unlinked data and linked data where limited hospitalisation history is available. This will allow translation of the study findings to the national setting to improve the accuracy of reporting of acute CHD trends. The developed methods will also be disseminated to the other Australian states and territories that have established linked data systems. Obtaining linked data at a national level is costly and time-consuming due 
to a lack of well-established and clear cross-jurisdictional protocols and permission processes for obtaining data. Hence, the methods we develop will be suitable for application by individual states to their own administrative data collections.

The algorithm developed in Objective 1 will therefore be modified and tested for use in other settings. It will first be applied to the national unlinked data sets, available through our collaboration on this project with the AIHW. We will also apply the algorithm to WA data using different scenarios of hospitalisation history to replicate linked data settings available in other jurisdictions (figure 1). This will inform us of the extent of agreement between the new validated algorithm and the other unlinked and linked data situations available in Australia. We will then systematically modify variables within the algorithm, including different lengths of lookback period (to exclude recurrent events), methods for identification of transfers, extent to which ED and biomarker data are included and aggregation of different subgroups of CHD. The extent of agreement between the best-practice algorithm and the modified versions will be tested by calculating the difference between each method in each calendar year. These data will be presented as temporal trends in the percentage of overestimation or underestimation of each algorithm, a method previously used by the AIHW. ${ }^{17}$ This will determine which modified version is the most accurate for use in unlinked data at a national level, and for linked data with limited history available.

Further specific issues which will be investigated are (1) the lack of concordance between coding of death in hospital and mortality register coding; (2) why some hospitalisations coded as 'transferred to another acute hospital' do not have a subsequent record identified in linked data; (3) ability to account for interstate transfers and (4) whether non-fatal acute CHD admissions should be included in the algorithm if they are transferred but not recorded as MI or unstable angina in the subsequent record (these cases are not included in the current national algorithm).

\section{Ethics and dissemination}

Approval to undertake this study has been provided by the Human Research Ethics Committees of the WA Department of Health (\#2016/23) and The University of Western Australia (RA/4/1/7230). The use of de-identified population-level linked data in Australia is provided under a waiver of consent, thereby removing the need for individual informed consent.

The monitoring algorithm developed by this project will be of great relevance for state-based and national monitoring and analyses of acute CHD in Australia. Dissemination of the new methods will be undertaken in conjunction with the AIHW, and will include workshops with representatives from relevant end-users of linked administrative health data in Australia. The findings will also have international relevance, as results will be of interest to researchers in international linked data jurisdictions. Results will be communicated to an international audience via peer-reviewed publications, reports and conference presentations.

\section{DISCUSSION}

Reliable and accurate monitoring of acute CHD is an important means of identifying changes in the epidemiology of this disease and demonstrating the impacts of acute care and primary and secondary prevention at the population level. Changes in diagnostic measures, clinical classifications and service-level factors over the past decade mean that previously applied methods for identifying MI and CHD in administrative health data, including distinguishing incident from recurrent events, are no longer reliable. Accurate measurement of trends in MI and other subgroups of CHD, which are indicators of severity and/or treatment approaches, will provide a better estimate of true population trends.

The findings of this study will have important implications for the monitoring of CHD in Australia and internationally. In Australia, the differing progress of data linkage systems means that different methods for monitoring may be required. For example, some jurisdictions have limited available hospitalisation history, or are unable to link multiple data collections. This also applies to international settings, where only unlinked data are available, or where shorter lookback periods are available in the linked data systems. Our methods and results may therefore be generalisable to some of these settings with comparable systems. The study will also have important clinical implications, as it will determine our ability to measure trends in STEMI and NSTEMI in a wholepopulation setting. Registry data are extremely important for reporting on patient characteristics, inhospital management and short and longer-term outcomes in these patient groups. However, they are seldom able to provide long-term temporal trends or whole-population data in this setting. The ability to measure accurate trends in STEMI and NSTEMI will provide important information on efficacy of treatment in each of these patients groups.

Rapid increases in presentation and admissions for chest pain have clearly had a major impact on the workload of EDs. ${ }^{27}$ Understanding the factors that have contributed to this, particularly the use of sensitive diagnostic tests, is essential for predicting future workloads and developing strategies to deal with the problem. Very little is known about the increasing population of persons who present each year to Australian hospitals with chest pain, but are not diagnosed with acute CHD. Comparison of the socio-demographic and clinical characteristics and long-term outcomes of those diagnosed with acute CHD will help identify patients in whom further follow-up may be required.

A major strength of this study is our ability to access data sources beyond morbidity and mortality data sets. The inclusion of clinical data, including ECGs and 


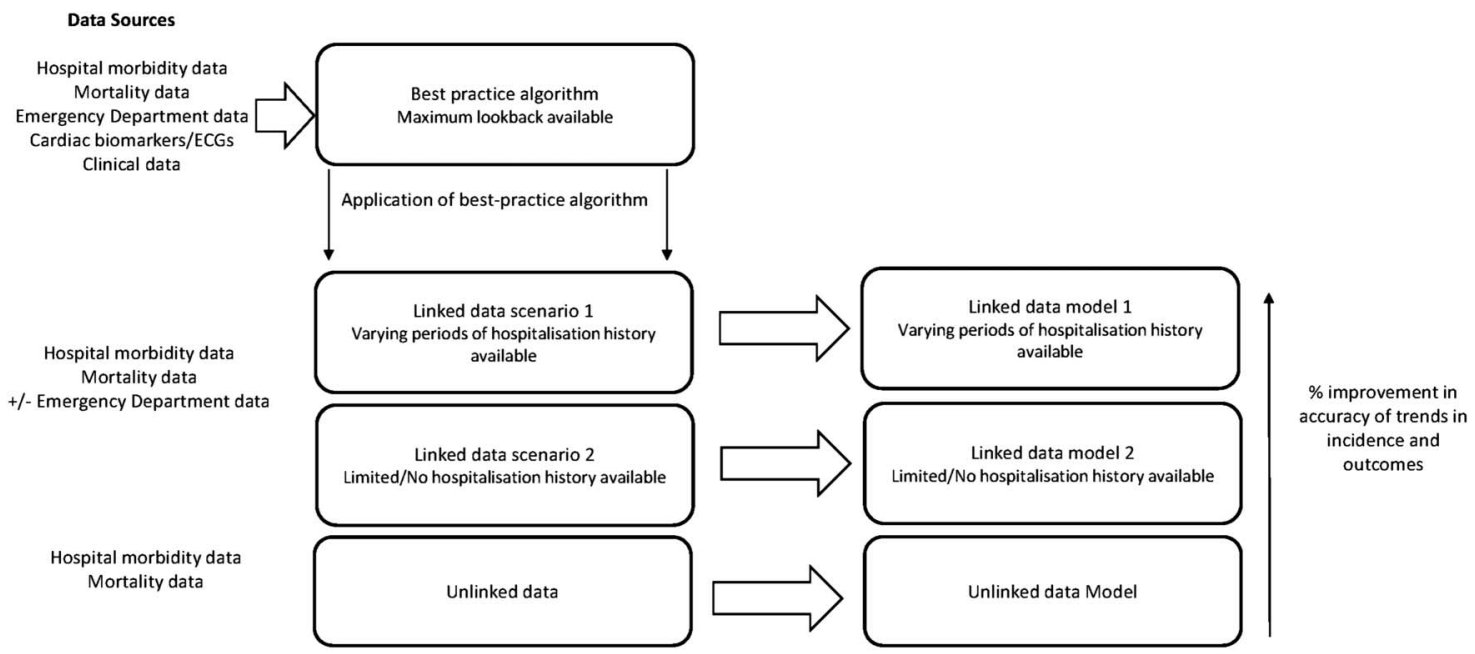

Figure 1 Flow chart of development of algorithms for measuring $\mathrm{CHD} /$ chest pain in administrative data according to different availability of data sources.

biomarker data in developing the monitoring algorithms, will improve the accuracy and utility of the algorithms, and will also broaden the scope of the use of administrative data. This will be a novel approach and ahead of current efforts to include laboratory data within linked data systems. This has the potential to extend to other linked data systems, particularly if the use of biomarker data substantially improves the monitoring algorithm. A further strength of the long-standing WADLS compared with newer state-based data linkage systems is the longterm availability of hospitalisation history for differentiating between incident and recurrent events, and the number of years available for trend analysis.

There are limitations in our study which are inherent with the use of administrative data sets. The analysis to determine the temporal consistency of recording of MI type within the administrative data will likely be based on reviewing cases coded as MI within the administrative data. Our previous validation work has already shown the proportion of cases which are misclassified and recorded as a non-MI code (usually unstable angina) ${ }^{22}$ and this will be taken into account in the analyses. Retrospective collection and review of ECGs from hospital records will only allow us to differentiate between STEMI and NSTEMI in terms of ECG criteria within clinical guidelines, but does not capture the complex setting within which clinicians are required to make diagnostic decisions. Thus, the use of biomarker data and other variables within the clinical and administrative data will also serve to underpin the analyses of temporal consistency. For the analyses including out-of-hospital deaths, we will be unable to validate cause of death coding from the original death certificates, but will instead test various algorithms, including use of multiple cause of death coding to enhance analyses. Additionally, we will only have information from administrative data to identify covariates, including comorbidities and procedures for the whole-population analyses (Objective 2), which can underestimate the prevalence of these variables. However, we have previously shown that there is a high sensitivity and specificity for the recording of diabetes in administrative data in CHD patients, ${ }^{15}$ and our previous use of clinical databases shows that coronary procedures are well recorded in our data sets. ${ }^{28}$

Although acute CHD, including MI and UA, is commonly managed in the hospital setting, there will be a proportion of cases managed in primary care or which remain undiagnosed. Additionally, hospitalisation data will not capture all stable and lower severity cases of CHD; therefore, our analyses of these CHD subgroups will be presented as hospitalisation rates rather than true whole-population rates of stable CHD.

\section{CONCLUSIONS}

An improved method for estimating population trends of the subgroups of acute CHD is required because of diagnostic and clinical changes that have occurred since 2000 in Australia. Calculating national and state rates based on subgroups of CHD using the methods we develop in this study will provide a better estimate of true population trends. The innovations from this study will advance the research utility of using administrative data beyond the advantages of the individual component data sets through the incorporation of data sources, including ED and cardiac biomarker data. Comparisons of outcomes between each of the subgroups will determine whether there are differences in risk, and whether there is benefit in monitoring these groups nationally.

\section{Author affiliations}

${ }^{1}$ School of Population Health, The University of Western Australia, Crawley, Western Australia, Australia

${ }^{2}$ Sir Charles Gairdner Hospital Unit, School of Medicine and Pharmacology (M503), The University of Western Australia, Crawley, Western Australia, Australia

${ }^{3}$ Australian Institute of Health and Welfare, Canberra, Australian Capital Territory, Australia 
${ }^{4}$ PathWest Laboratory Medicine WA, Sir Charles Gairdner Hospital, Nedlands, Western Australia, Australia

${ }^{5}$ School of Pathology and Laboratory Medicine, The University of Western Australia, Perth, Western Australia, Australia

Twitter Follow Frank Sanfilippo at @CVRG_UWA

Acknowledgements The authors thank staff at the Western Australian Data Linkage Branch (WA Department of Health), the Department of Health Inpatient Data Collections and Registrar General for provision of the linked data which will be used in this study.

Contributors The study was initially conceived by MSTH and FMS. FMS wrote the initial draft of the project grant application, with all investigators contributing to the study design and methodology. LN wrote the study protocol manuscript with scientific input from all coauthors. MK provided advice on statistical analysis and sample size calculations; JB advised on pathology data and analysis of biomarker data; $\mathrm{JH}$ provided clinical input and advice on medical record reviews; FMS, LN, MSTH, MK, TGB, DL, SM and AR provided input on methodology and planning of analyses.

Funding This study is supported by a project grant from the National Health and Medical Research Council (NHMRC) of Australia (grant \#1078978). LN is supported by an NHMRC Early Career Fellowship.

Competing interests None declared.

Ethics approval Department of Health WA and The University of Western Australia Human Research Ethics Committee.

Provenance and peer review Not commissioned; externally peer reviewed.

Data sharing statement The authors will consider requests for data sharing on an individual basis. However, this project uses third-party data derived from State government registries, which are ultimately governed by their ethics committees and data custodians. Therefore, any requests to share these data will be subject to formal approval from their ethics committees overseeing the use of these data sources, along with the data custodians for the data of interest.

Open Access This is an Open Access article distributed in accordance with the Creative Commons Attribution Non Commercial (CC BY-NC 4.0) license which permits others to distribute, remix, adapt, build upon this work noncommercially, and license their derivative works on different terms, provided the original work is properly cited and the use is non-commercial. See: http:// creativecommons.org/licenses/by-nc/4.0/

\section{REFERENCES}

1. Beaglehole R. International trends in coronary heart disease mortality and incidence rates. J Cardiovasc Risk 1999;6:63-8.

2. Australian Institute of Health and Welfare. Australian burden of disease study: impact and causes of illness and death in Australia 2011. Canberra: AlHW, 2016. Australian Burden of Disease Study Series no. 3 BOD 4.

3. Jamrozik K, Dobson AJ, Hobbs MS, et al. Monitoring the incidence of cardiovascular disease in Australia. Canberra: AlHW, 2001. AlHW cat. no. CVD 16.

4. Sanfilippo FM, Hobbs MST, Knuiman MW, et al. Impact of new biomarkers of myocardial damage on trends in myocardial infarction hospital admission rates from population-based administrative data. Am J Epidemiol 2008;168:225-33.

5. Roger VL, Weston SA, Gerber Y, et al. Trends in incidence, severity and outcome of hospitalized myocardial infarction. Circulation 2010;121:863-9.

6. Alpert JS, Thygesen K, Antman E, et al. Myocardial infarction redefined-a consensus document of The Joint European Society of Cardiology/American College of Cardiology committee for the redefinition of myocardial infarction. J Am Coll Cardiol 2000;36:959-69.

7. Chew DP, Aroney CN, Aylward PE, et al. 2011 Addendum to The National Heart Foundation of Australia/Cardiac Society of Australia and New Zealand guidelines for the management of acute coronary syndromes (ACS) 2006. Heart Lung Circ 2011;20:487-502.

8. Rosamond WD, Chambless LE, Heiss G, et al. Twenty-two-year trends in incidence of myocardial infarction, coronary heart disease mortality, and case fatality in 4 US communities, 1987-2008. Circulation 2012;125:1848-57.

9. Cannon CP. Update to International Classification of Diseases, 9th revision codes: distinguishes STEMI from NSTEMI. Crit Pathw Cardiol 2005;4:185-6.

10. Yeh RW, Sidney S, Chandra M, et al. Population trends in the incidence and outcomes of acute myocardial infarction. N Eng J Med 2010;362:2155-65.

11. Jennings SM, Bennett $\mathrm{K}$, Lonergan $\mathrm{M}$, et al. Trends in hospitalisation for acute myocardial infarction in Ireland, 1997-2008. Heart 2012;98:1285-9.

12. Australian Institute of Health and Welfare. Monitoring acute coronary syndrome using national hospital data: an information paper on trends and issues. Canberra: AlHW, 2011. Cat. no. CVD 57.

13. Nedkoff LJ, Briffa TG, Preen DB, et al. Age- and sex-specific trends in the incidence of hospitalized acute coronary syndromes in Western Australia. Circ Cardiovasc Qual Outcomes 2011;4:557-64.

14. Katzenellenbogen JM, Sanfilippo FM, Hobbs MST, et al. Aboriginal to non-Aboriginal differentials in 2-year outcomes following non-fatal first-ever acute MI persist after adjustment for comorbidity. Eur J Prev Cardiol 2012;19:983-90.

15. Nedkoff L, Knuiman M, Hung J, et al. Concordance between administrative health data and medical records for diabetes status in coronary heart disease patients: a retrospective linked data study. BMC Med Res Methodol 2013;13:121.

16. Lopez D, Katzenellenbogen JM, Sanfilippo FM, et al. Transfers to metropolitan hospitals and coronary angiography for rural Aboriginal and non-Aboriginal patients with acute ischaemic heart disease in Western Australia. BMC Cardiovasc Disord 2014;14:58.

17. Australian Institute of Health and Welfare. Acute coronary syndrome: validation of the method used to monitor incidence in Australia. A working paper using linked hospitalisation and deaths data from Western Australia and New South Wales. Canberra: AIHW, 2014. CVD 68.

18. Australian Bureau of Statistics (ABS). 3101.0 Australian Demographic Statistics, December 2015. 2015. http://abs.gov.au/ AUSSTATS/abs@.nsf/DetailsPage/3101.0Dec\%202015? OpenDocument (accessed online Aug 2016).

19. Clark A, Preen $\mathrm{DB}, \mathrm{Ng} \mathrm{JQ}$, et al. Is Western Australia representative of other Australian States and Territories in terms of key socio-demographic and health economic indicators? Aust Health Rev 2010;34:210-15

20. Holman CDJ, Bass AJ, Rouse IL, et al. Population-based linkage of health records in Western Australia: development of a health services research linked database. Aust N Z J Public Health 1999;23:453-9.

21. Australian Institute of Health and Welfare. Multiple causes of death: an analysis of all natural and selected chronic disease causes of death 1997-2007. Canberra: AlHW, 2012. Bulletin no. 105. Cat no. AUS 159

22. Sanfilippo FM, Hobbs MST, Knuiman MW, et al. Can we monitor heart attack in the troponin era: evidence from a population-based cohort study. BMC Cardiovasc Disord 2011;11:35.

23. Luepker RV, Apple FS, Christenson RH, et al., AHA Council on Epidemiology and Prevention; AHA Statistics Committee; World Heart Federation Council on Epidemiology and Prevention, et al. Case definitions for acute coronary heart disease in epidemiology and clinical research studies: a statement from the AHA Council on Epidemiology and Prevention; AHA Statistics Committee; World Heart Federation Council on Epidemiology and Prevention; the European Society of Cardiology Working Group on Epidemiology and Prevention; Centers for Disease Control and Prevention; and The National Heart, Lung, and Blood Institute. Circulation 2003;108:2543-9.

24. Thygesen K, Alpert JS, Jaffe AS. 2013 ACCF/AHA guideline for the management of ST-elevation myocardial infarction. A report of the American College of Cardiology Foundation/American Heart Association Task Force on Practice Guidelines. J Am Coll Cardiol 2012;126:2020-35.

25. Amsterdam EA, Wenger NK, Brindis RG, et al. 2014 AHA/ACC guideline for the management of patients with non-ST-elevation acute coronary syndromes: a report of the American College of Cardiology/American Heart Association Task Force on Practice Guidelines. J Am Coll Cardiol 2014;64:e139-228.

26. Canadian Institute for Health Information. Hospital care for heart attacks among First Nations, Inuit and Metis. Canada: Canadian Institute for Health Information, 2013.

27. Australian Institute of Health and Welfare. Australian hospital statistics 2011-12: emergency department care. Canberra: AlHW, 2012. Cat. no. HSE 126.

28. Sanfilippo FM, Rankin JM, Hobbs MST, et al. Impact of the introduction of drug eluting stents on clinical outcomes in patients undergoing percutaneous and surgical coronary artery revascularisation procedures in Western Australia. BMC Cardiovasc Disord 2013;13:47. 